\title{
A general iterative algorithm for an infinite family of nonexpansive operators in Hilbert spaces
}

Cuijie Zhang ${ }^{*}$ and Songnian He

"Correspondence:

zhang_cui_jie@126.com

College of Science, Civil Aviation

University of China, Tianjin, 300300,

P.R. China

\begin{abstract}
In this paper, we introduce a new general iterative algorithm for an infinite family of nonexpansive operators in Hilbert spaces. Under suitable assumptions, we prove that the sequence generated by the iterative algorithm converges strongly to a common point of the sets of fixed points, which solves a variational inequality. Our results improve and extend the corresponding results announced by many others. As applications, at the end of the paper, we apply our results to the split common fixed point problem.
\end{abstract}

Keywords: an infinite family of nonexpansive operators; strong convergence; $k$-Lipschitizian; $\eta$-strongly monotone; split common fixed point problem

\section{Introduction}

Let $H$ be a real Hilbert space with the inner product $\langle\cdot, \cdot \cdot\rangle$ and the norm $\|\cdot\|$. Let $T$ be a nonexpansive operator. The set of fixed points of $T$ is denoted by Fix $(T)$. In 2000, Moudafi [1] introduced the viscosity approximation method for a nonexpansive operator and considered the sequence $\left\{x_{n}\right\}$ by

$$
x_{n+1}=\alpha_{n} f x_{n}+\left(1-\alpha_{n}\right) T x_{n},
$$

where $f$ is a contraction on $H$ and $\left\{\alpha_{n}\right\}$ is a sequence in $(0,1)$. In 2004, $\mathrm{Xu}$ [2] proved that under some conditions on $\left\{\alpha_{n}\right\}$, the sequence $\left\{x_{n}\right\}$ generated by (1.1) strongly converges to $x^{*}$ in $\operatorname{Fix}(T)$ which is the unique solution of the variational inequality

$$
\left\langle(I-f) x^{*}, x-x^{*}\right\rangle \geq 0, \quad \forall x \in \operatorname{Fix}(T) .
$$

It is well known that iterative methods for nonexpansive operators have been used to solve convex minimization problems; see, e.g., [3, 4]. A typical problem is to minimize a quadratic function over the set of fixed points of a nonexpansive operator $T$ on a real Hilbert space $H$ :

$$
\min _{x \in \operatorname{Fix}(T)} \frac{1}{2}\langle A x, x\rangle-\langle x, b\rangle,
$$

\section{Springer}

(C) 2013 Zhang and He; licensee Springer. This is an Open Access article distributed under the terms of the Creative Commons Attribution License (http://creativecommons.org/licenses/by/2.0), which permits unrestricted use, distribution, and reproduction in any medium, provided the original work is properly cited. 
where $b$ is a given point in $H$ and $A$ is a strongly positive bounded linear operator. In [3], $\mathrm{Xu}$ proved that the sequence $\left\{x_{n}\right\}$ defined by the following iterative method:

$$
x_{n+1}=\left(I-\alpha_{n} A\right) T x_{n}+\alpha_{n} b \text {, }
$$

converges strongly to the unique solution of the minimization problem (1.2). In [5], Marino and $\mathrm{Xu}$ combined the iterative method (1.3) and the viscosity method (1.1) and considered the following general iterative method:

$$
x_{n+1}=\alpha_{n} \gamma f x_{n}+\left(I-\alpha_{n} A\right) T x_{n} .
$$

They proved that the sequence $\left\{x_{n}\right\}$ generated by (1.4) converges strongly to the unique solution of the variational inequality

$$
\left\langle(A-\gamma f) x^{*}, x-x^{*}\right\rangle \geq 0, \quad \forall x \in \operatorname{Fix}(T),
$$

which is the optimality condition for the minimization problem

$$
\min _{x \in \operatorname{Fix}(T)} \frac{1}{2}\langle A x, x\rangle-h(x)
$$

where $h$ is a potential function for $\gamma f$ (i.e., $h^{\prime}(x)=\gamma f(x)$ for $x \in H$ ).

On the other hand, Yamada [4] in 2001 introduced the following hybrid iterative method:

$$
x_{n+1}=T x_{n}-\mu \lambda_{n} F T x_{n}, \quad n \geq 0,
$$

where $F$ is a $k$-Lipschitzian and $\eta$-strongly monotone operator with $k>0, \eta>0$ and $0<\mu<2 \eta / k^{2}$. Under some appropriate conditions, he proved that the sequence $\left\{x_{n}\right\}$ generated by (1.5) converges strongly to the unique solution of the variational inequality

$$
\langle F \tilde{x}, x-\tilde{x}\rangle \geq 0, \quad \forall x \in \operatorname{Fix}(T)
$$

Recently, combining (1.4) and (1.5), Tian [6] considered the following general iterative method:

$$
x_{n+1}=\alpha_{n} \gamma f x_{n}+\left(I-\mu \alpha_{n} F\right) T x_{n} .
$$

Improving and extending the corresponding results given by Marino, $\mathrm{Xu}$ and Yamada, he proved that the sequence $\left\{x_{n}\right\}$ generated by (1.6) converges strongly to the unique solution $x^{*} \in \operatorname{Fix}(T)$ of the variational inequality

$$
\langle(\gamma f-\mu F) \tilde{x}, x-\tilde{x}\rangle \leq 0, \quad \forall x \in \operatorname{Fix}(T)
$$

Based on the above results of Marino, Xu, Yamada and Tian, much generalization work has been made by the corresponding authors; for instance, [7-23]. The problem of finding an element in the intersection of the fixed point sets of an infinite family of nonexpansive 
operators has attracted much attention because of its extraordinary utility and broad applicability in many branches of mathematical science and engineering. For example, if the nonexpansive operators are projection onto some closed convex sets $C_{i}(i \in \mathbb{N})$ in a real Hilbert space $H$, then such a fixed point problem becomes the convex feasibility problem of finding a point in $\bigcap_{i \in \mathbb{N}} C_{i}$. Many previous results [24-31] and many results not cited here considered the common fixed point about an infinite family of nonexpansive operators by $W_{n}$-mappings.

Motivated and inspired by the above results, we consider the following iterative algorithm without $W_{n}$-mappings:

$$
\left\{\begin{array}{l}
y_{n}=\beta_{n} x_{n}+\sum_{i=1}^{n}\left(\beta_{i-1}-\beta_{i}\right) T_{i} x_{n}, \\
x_{n+1}=\alpha_{n} \gamma V x_{n}+\left(I-\mu \alpha_{n} F\right) y_{n},
\end{array}\right.
$$

where $\left\{\alpha_{n}\right\}$ is a sequence in $(0,1]$ and $\left\{\beta_{n}\right\}$ is a strictly decreasing sequence in $(0,1]$. Under some appropriate conditions, we proved the sequence $\left\{x_{n}\right\}$ generated by (1.7) converges strongly to the unique solution of the variational inequality:

$$
\langle(\mu F-\gamma V) \tilde{x}, z-\tilde{x}\rangle \geq 0, \quad \forall z \in \bigcap_{i=1}^{\infty} \operatorname{Fix}\left(T_{i}\right) .
$$

Our results improve and extend the corresponding results announced by many others. As applications, at the end of the paper, we apply our results to the split common fixed point problem.

\section{Preliminaries}

Throughout this paper, we write $x_{n} \rightarrow x$ and $x_{n} \rightarrow x$ to indicate that $\left\{x_{n}\right\}$ converges weakly to $x$ and converges strongly to $x$, respectively.

An operator $T: H \rightarrow H$ is said to be nonexpansive if $\|T x-T y\| \leq\|x-y\|$ for all $x, y \in H$. It is well known that $\operatorname{Fix}(T)$ is closed and convex. It is known that $A$ is called strongly positive if there exists a constant $\gamma>0$ such that $\langle A x, x\rangle \geq \gamma\|x\|^{2}$ for all $x \in H$. The operator $F$ is called $\eta$-strongly monotone if there exists a constant $\eta>0$ such that

$$
\langle x-y, F x-F y\rangle \geq \eta\|x-y\|^{2}
$$

for all $x, y \in H$.

In order to prove our main results, we collect the following lemmas in this section.

Lemma 2.1 (Demiclosedness principle [32]) Let H be a Hilbert space, C be a closed convex subset of $H$, and $T: C \rightarrow C$ be a nonexpansive operator with $\operatorname{Fix}(T) \neq \emptyset$. If $\left\{x_{n}\right\}$ is a sequence in $C$ weakly converging to $x \in C$ and $\left\{(I-T) x_{n}\right\}$ converges strongly to $y \in C$, then $(I-T) x=y$. In particular, if $y=0$, then $x \in \operatorname{Fix}(T)$.

Lemma 2.2 [2] Assume that $\left\{a_{n}\right\}$ is a sequence of nonnegative real numbers such that

$$
a_{n+1} \leq\left(1-\gamma_{n}\right) a_{n}+\delta_{n}, \quad n \geq 0,
$$

where $\left\{\gamma_{n}\right\}$ is a sequence in $(0,1)$ and $\left\{\delta_{n}\right\}$ is a sequence such that 
(i) $\sum_{n=1}^{\infty} \gamma_{n}=\infty$,

(ii) $\lim \sup _{n \rightarrow \infty} \frac{\delta_{n}}{\gamma_{n}} \leq 0$ or $\sum_{n=1}^{\infty}\left|\delta_{n}\right|<\infty$.

Then $\lim _{n \rightarrow \infty} a_{n}=0$.

Lemma 2.3 [33] Let $H$ be a real Hilbert space, let $V: H \rightarrow H$ be an L-Lipschitzian operator with $L>0$, and let $F: H \rightarrow H$ be a $k$-Lipschitzian continuous operator and $\eta$-strongly monotone operator with $k>0, \eta>0$. Then, for $0<\gamma<\frac{\mu \eta}{L}, \mu F-\gamma V$ is strongly monotone with coefficient $\mu \eta-\gamma L$.

Lemma 2.4 [34] Let $C$ be a closed convex subset of a real Hilbert space $H$, given $x \in H$ and $y \in C$. Then $y=P_{C} x$ if and only if the following inequality holds:

$$
\langle x-y, z-y\rangle \leq 0
$$

for every $z \in C$.

\section{Main results}

Lemma 3.1 Let $\left\{T_{n}\right\}: H \rightarrow H$ be an infinite family of nonexpansive operators, let $F: H \rightarrow$ $H$ be a $k$-Lipschitzian and $\eta$-strongly monotone operator with $k>0$ and $\eta>0$, and let $V: H \rightarrow H$ be an L-Lipschitzian operator. Let $0<\mu<\frac{2 \eta}{k^{2}}$ and $0<\gamma<\frac{\mu\left(\eta-\frac{1}{2} \mu k^{2}\right)}{L}=\frac{\tau}{L}$. Assume that $S_{n}=\beta_{n} I+\sum_{i=1}^{n}\left(\beta_{i-1}-\beta_{i}\right) T_{i}$, where $\left\{\beta_{n}\right\}$ is a strictly decreasing sequence with $\beta_{0}=1$ and $\beta_{n} \in(0,1]$. Consider the following mapping $G_{n}$ on $H$ defined by

$$
G_{n} x=\alpha_{n} \gamma V x+\left(I-\mu \alpha_{n} F\right) S_{n} x
$$

where $\left\{\alpha_{n}\right\}$ is a sequence in $(0,1]$. Then $G_{n}$ is a contraction.

Proof Observe that

$$
\begin{aligned}
\left\|G_{n} x-G_{n} y\right\| & \leq \alpha_{n} \gamma\|V x-V y\|+\left(1-\alpha_{n} \tau\right)\left\|S_{n} x-S_{n} y\right\| \\
& \leq \alpha_{n} \gamma L\|x-y\|+\left(1-\alpha_{n} \tau\right)\left\|\beta_{n}(x-y)+\sum_{i=1}^{n}\left(\beta_{i-1}-\beta_{i}\right)\left(T_{i} x-T_{i} y\right)\right\| \\
& \leq \alpha_{n} \gamma L\|x-y\|+\left(1-\alpha_{n} \tau\right)\left(\beta_{n}\|x-y\|+\sum_{i=1}^{n}\left(\beta_{i-1}-\beta_{i}\right)\|x-y\|\right) \\
& =\alpha_{n} \gamma L\|x-y\|+\left(1-\alpha_{n} \tau\right)\|x-y\| \\
& =\left(1-\alpha_{n}(\tau-\gamma L)\right)\|x-y\| .
\end{aligned}
$$

Since $0<1-\alpha_{n}(\tau-\gamma L)<1, G_{n}$ is a contraction. This completes the proof.

Since $G_{n}$ is a contraction, using the Banach contraction principle, $G_{n}$ has a unique fixed point $x_{n}^{V} \in H$ such that

$$
x_{n}^{V}=\alpha_{n} \gamma V x_{n}^{V}+\left(I-\mu \alpha_{n} F\right) S_{n} x_{n}^{V} .
$$

For simplicity, we denote $x_{n}$ for $x_{n}^{V}$ without confusion.

Now we state and prove our main results in this paper. 
Theorem 3.2 Let $\left\{T_{n}\right\}$ be an infinite family of nonexpansive self-mappings of a real Hilbert space $H$, let $F$ be a $k$-Lipschitzian and $\eta$-strongly monotone operator on $H$ with $k>0$ and $\eta>0$, and let $V$ be an L-Lipschitzian operator. Suppose that $\Omega=\bigcap_{i=1}^{\infty} \operatorname{Fix}\left(T_{i}\right)$ is nonempty. Suppose that $\left\{x_{n}\right\}$ is generated by the following algorithm:

$$
\left\{\begin{array}{l}
y_{n}=\beta_{n} x_{n}+\sum_{i=1}^{n}\left(\beta_{i-1}-\beta_{i}\right) T_{i} x_{n}, \\
x_{n}=\alpha_{n} \gamma V x_{n}+\left(I-\mu \alpha_{n} F\right) y_{n},
\end{array}\right.
$$

where $0<\mu<\frac{2 \eta}{k^{2}}$ and $0<\gamma<\frac{\tau}{L}$ with $\tau=\mu\left(\eta-\frac{1}{2} \mu k^{2}\right)$. If the following conditions are satisfied:

(i) $\left\{\alpha_{n}\right\}$ is a sequence in $(0,1]$ and $\lim _{n \rightarrow \infty} \alpha_{n}=0$;

(ii) $\left\{\beta_{n}\right\}$ is a strictly decreasing sequence in $(0,1]$ and $\beta_{0}=1$.

Then $\left\{x_{n}\right\}$ converges strongly to $\tilde{x} \in \Omega$, which solves the variational inequality:

$$
\langle(\mu F-\gamma V) \tilde{x}, z-\tilde{x}\rangle \geq 0, \quad \forall z \in \Omega
$$

Equivalently, we have $P_{\Omega}(I-\mu F+\gamma V) \tilde{x}=\tilde{x}$.

Proof We proceed with the following steps:

Step 1: First we show that $\left\{x_{n}\right\}$ is bounded.

In fact, let $p \in \Omega$, then for every $i \in \mathbb{N}, T_{i} p=p$. Observe that

$$
\left\|y_{n}-p\right\| \leq \beta_{n}\left\|x_{n}-p\right\|+\sum_{i=1}^{n}\left(\beta_{i-1}-\beta_{i}\right)\left\|T_{i} x_{n}-T_{i} p\right\| \leq\left\|x_{n}-p\right\| .
$$

Thus it follows that

$$
\begin{aligned}
\left\|x_{n}-p\right\| & =\left\|\alpha_{n} \gamma V x_{n}+\left(I-\mu \alpha_{n} F\right) y_{n}-p\right\| \\
& =\left\|\alpha_{n}\left(\gamma V x_{n}-\mu F p\right)+\left(I-\mu \alpha_{n} F\right) y_{n}-\left(I-\mu \alpha_{n} F\right) p\right\| \\
& \leq\left(1-\alpha_{n} \tau\right)\left\|y_{n}-p\right\|+\alpha_{n}\left(\left\|\gamma V x_{n}-\gamma V p\right\|+\|\gamma V p-\mu F p\|\right) \\
& \leq\left(1-\alpha_{n} \tau\right)\left\|y_{n}-p\right\|+\alpha_{n} \gamma L\left\|x_{n}-p\right\|+\alpha_{n}\|\gamma V p-\mu F p\| \\
& \leq\left(1-\alpha_{n}(\tau-\gamma L)\right)\left\|x_{n}-p\right\|+\alpha_{n}\|\gamma V p-\mu F p\| .
\end{aligned}
$$

Then we have

$$
\left\|x_{n}-p\right\| \leq \frac{1}{\tau-\gamma L}\|\gamma V p-\mu F p\|,
$$

which implies that $\left\{x_{n}\right\}$ is bounded. Hence we can obtain $\left\{y_{n}\right\},\left\{T_{i} x_{n}\right\},\left\{F y_{n}\right\}$ and $\left\{V x_{n}\right\}$ are bounded. Note that

$$
\left\|x_{n}-y_{n}\right\|=\left\|\alpha_{n} \gamma V x_{n}+\left(I-\mu \alpha_{n} F\right) y_{n}-y_{n}\right\|=\alpha_{n}\left\|\gamma V x_{n}-\mu F y_{n}\right\|,
$$

we immediately obtain that

$$
\lim _{n \rightarrow \infty}\left\|x_{n}-y_{n}\right\|=0 .
$$


Step 2: We show $\lim _{n \rightarrow \infty}\left\|x_{n}-T_{i} x_{n}\right\|=0$.

Since $p \in \Omega$, we note that

$$
\begin{aligned}
\left\|x_{n}-p\right\|^{2} & \geq\left\|T_{i} x_{n}-p\right\|^{2}=\left\|T_{i} x_{n}-x_{n}+x_{n}-p\right\|^{2} \\
& =\left\|T_{i} x_{n}-x_{n}\right\|^{2}+\left\|x_{n}-p\right\|^{2}+2\left\langle T_{i} x_{n}-x_{n}, x_{n}-p\right\rangle,
\end{aligned}
$$

which implies that

$$
\frac{1}{2}\left\|T_{i} x_{n}-x_{n}\right\|^{2} \leq\left\langle x_{n}-T_{i} x_{n}, x_{n}-p\right\rangle .
$$

Thus

$$
\begin{aligned}
\frac{1}{2} \sum_{i=1}^{n}\left(\beta_{i-1}-\beta_{i}\right)\left\|T_{i} x_{n}-x_{n}\right\|^{2} & \leq \sum_{i=1}^{n}\left(\beta_{i-1}-\beta_{i}\right)\left\langle x_{n}-T_{i} x_{n}, x_{n}-p\right\rangle \\
& =\left\langle\left(1-\beta_{n}\right) x_{n}-\sum_{i=1}^{n}\left(\beta_{i-1}-\beta_{i}\right) T_{i} x_{n}, x_{n}-p\right\rangle \\
& =\left\langle\left(1-\beta_{n}\right) x_{n}-y_{n}+\beta_{n} x_{n}, x_{n}-p\right\rangle \\
& =\left\langle x_{n}-y_{n}, x_{n}-p\right\rangle \\
& \leq\left\|x_{n}-y_{n}\right\|\left\|x_{n}-p\right\| .
\end{aligned}
$$

Then we immediately obtain $\lim _{n \rightarrow \infty} \sum_{i=1}^{n}\left(\beta_{i-1}-\beta_{i}\right)\left\|T_{i} x_{n}-x_{n}\right\|^{2}=0$. Since $\left\{\beta_{n}\right\}$ is strictly decreasing, it follows that

$$
\lim _{n \rightarrow \infty}\left\|T_{i} x_{n}-x_{n}\right\|=0
$$

for every $i \in \mathbb{N}$. Since $S_{n}=\beta_{n} I+\sum_{i=1}^{n}\left(\beta_{i-1}-\beta_{i}\right) T_{i}$, thus

$$
\left\|x_{n}-S_{n} x_{n}\right\|^{2} \leq \sum_{i=1}^{n}\left(\beta_{i-1}-\beta_{i}\right)\left\|T_{i} x_{n}-x_{n}\right\|^{2} .
$$

It shows that

$$
\lim _{n \rightarrow \infty}\left\|x_{n}-S_{n} x_{n}\right\|=0
$$

Step 3: We show that there exists a subsequence $\left\{x_{n_{k}}\right\}$ of $\left\{x_{n}\right\}$ such that $x_{n_{k}} \rightarrow \tilde{x}$.

Since $\left\{x_{n}\right\}$ is bounded, there exist a point $\tilde{x} \in H$ and a subsequence $\left\{x_{n_{k}}\right\}$ of $\left\{x_{n}\right\}$ such that $x_{n_{k}} \rightarrow \tilde{x}$. By Lemma 2.1 and (3.4), we obtain $\tilde{x} \in \operatorname{Fix}\left(T_{i}\right)$ for any $i \in \mathbb{N}$. This shows that $\tilde{x} \in \Omega$. On the other hand, we note that

$$
\begin{aligned}
x_{n}-\tilde{x}= & \alpha_{n} \gamma V x_{n}+\left(I-\mu \alpha_{n} F\right) y_{n}-\tilde{x} \\
= & \left(I-\alpha_{n}(\mu F-\gamma V)\right) y_{n}-\left(I-\alpha_{n}(\mu F-\gamma V)\right) \tilde{x} \\
& -\alpha_{n}(\mu F-\gamma V) \tilde{x}+\alpha_{n}\left(\gamma V x_{n}-\gamma V y_{n}\right) .
\end{aligned}
$$


Hence we obtain

$$
\begin{aligned}
\left\|x_{n}-\tilde{x}\right\|^{2}= & \left\langle\left(I-\alpha_{n}(\mu F-\gamma V)\right) y_{n}-\left(I-\alpha_{n}(\mu F-\gamma V)\right) \tilde{x}, x_{n}-\tilde{x}\right\rangle \\
& -\alpha_{n}\left\langle(\mu F-\gamma V) \tilde{x}, x_{n}-\tilde{x}\right\rangle+\alpha_{n}\left\langle\gamma V x_{n}-\gamma V y_{n}, x_{n}-\tilde{x}\right\rangle \\
\leq & \left\|\left(I-\alpha_{n}(\mu F-\gamma V)\right) y_{n}-\left(I-\alpha_{n}(\mu F-\gamma V)\right) \tilde{x}\right\|\left\|x_{n}-\tilde{x}\right\| \\
& -\alpha_{n}\left\langle(\mu F-\gamma V) \tilde{x}, x_{n}-\tilde{x}\right\rangle+\alpha_{n} \gamma L\left\|x_{n}-y_{n}\right\|\left\|x_{n}-\tilde{x}\right\| \\
\leq & \left(1-\alpha_{n}(\tau-\gamma L)\right)\left\|x_{n}-\tilde{x}\right\|^{2}-\alpha_{n}\left\langle(\mu F-\gamma V) \tilde{x}, x_{n}-\tilde{x}\right\rangle \\
& +\alpha_{n} \gamma L\left\|x_{n}-y_{n}\right\|\left\|x_{n}-\tilde{x}\right\| .
\end{aligned}
$$

Then it follows that

$$
\left\|x_{n}-\tilde{x}\right\|^{2} \leq \frac{\gamma L}{\tau-\gamma L}\left\|x_{n}-y_{n}\right\|\left\|x_{n}-\tilde{x}\right\|-\frac{1}{\tau-\gamma L}\left\langle(\mu F-\gamma V) \tilde{x}, x_{n}-\tilde{x}\right\rangle .
$$

In particular,

$$
\left\|x_{n_{k}}-\tilde{x}\right\|^{2} \leq \frac{\gamma L}{\tau-\gamma L}\left\|x_{n_{k}}-y_{n_{k}}\right\|\left\|x_{n_{k}}-\tilde{x}\right\|-\frac{1}{\tau-\gamma L}\left\langle(\mu F-\gamma V) \tilde{x}, x_{n_{k}}-\tilde{x}\right\rangle .
$$

From $x_{n_{k}} \rightarrow \tilde{x}$ and (3.3), it follows that $x_{n_{k}} \rightarrow \tilde{x}$.

Step 4: We show that $\tilde{x}$ solves the variational inequality (3.2).

Observe that

$$
x_{n}=\alpha_{n} \gamma V x_{n}+\left(I-\mu \alpha_{n} F\right) S_{n} x_{n} .
$$

Hence, we conclude that

$$
\begin{aligned}
(\mu F-\gamma V) x_{n} & =(\mu F-\gamma V)\left(x_{n}-S_{n} x_{n}\right)+\mu F S_{n} x_{n}-\gamma V S_{n} x_{n} \\
& =(\mu F-\gamma V)\left(x_{n}-S_{n} x_{n}\right)+\left(\gamma V x_{n}-\gamma V S_{n} x_{n}\right)-\gamma V x_{n}+\mu F S_{n} x_{n} \\
& =(\mu F-\gamma V)\left(x_{n}-S_{n} x_{n}\right)+\left(\gamma V x_{n}-\gamma V S_{n} x_{n}\right)-\frac{1}{\alpha_{n}}\left(I-S_{n}\right) x_{n} .
\end{aligned}
$$

Since $S_{n}$ is nonexpansive, we have that $I-S_{n}$ is monotone. Note that for any $z \in \Omega, S_{n} z=z$. Then we deduce

$$
\begin{aligned}
\left\langle(\mu F-\gamma V) x_{n}, x_{n}-z\right\rangle= & -\frac{1}{\alpha_{n}}\left\langle\left(I-S_{n}\right) x_{n}-\left(I-S_{n}\right) z, x_{n}-z\right\rangle \\
& +\left\langle(\mu F-\gamma V)\left(I-S_{n}\right) x_{n}, x_{n}-z\right\rangle+\left\langle\gamma V x_{n}-\gamma V S_{n} x_{n}, x_{n}-z\right\rangle \\
\leq & \left\langle(\mu F-\gamma V)\left(I-S_{n}\right) x_{n}, x_{n}-z\right\rangle+\gamma L\left\|x_{n}-S_{n} x_{n}\right\|\left\|x_{n}-z\right\| .
\end{aligned}
$$

Now, replacing $n$ with $n_{k}$ in the above inequality, and letting $k \rightarrow \infty$, by (3.5) we have

$$
\begin{aligned}
\langle(\mu F-\gamma V) \tilde{x}, \tilde{x}-z\rangle & =\lim _{k \rightarrow 0}\left\langle(\mu F-\gamma V) x_{n_{k}}, x_{n_{k}}-z\right\rangle \\
& \leq \lim _{k \rightarrow 0}\left(\left\langle(\mu F-\gamma V)\left(x_{n_{k}}-S_{n_{k}} x_{n_{k}}\right), x_{n_{k}}-z\right\rangle\right.
\end{aligned}
$$




$$
\begin{aligned}
& \left.+\gamma L\left\|x_{n_{k}}-S_{n_{k}} x_{n_{k}}\right\|\left\|x_{n_{k}}-z\right\|\right) \\
= & 0 .
\end{aligned}
$$

That is, $\langle(\mu F-\gamma V) \tilde{x}, z-\tilde{x}\rangle \geq 0$ for every $z \in \Omega$. It follows that $\tilde{x}$ is a solution of the variational inequality (3.2). Since $\mu F-\gamma V$ is $(\mu \eta-\gamma L)$-strongly monotone and $(\mu k-\gamma L)$ Lipschitzian, the variational inequality (3.2) has a unique solution. So, we conclude that $x_{n} \rightarrow \tilde{x}$ as $n \rightarrow \infty$. The variational inequality (3.2) can be written as

$$
\langle(I-\mu F+\gamma V) \tilde{x}-\tilde{x}, z-\tilde{x}\rangle \leq 0, \quad \forall z \in \Omega
$$

By Lemma 2.4, we have $P_{\Omega}(I-\mu F+\gamma V) \tilde{x}=\tilde{x}$.

Theorem 3.3 Let $\left\{T_{n}\right\}$ be an infinite family of nonexpansive self-mappings of a real Hilbert space $H$, let $F$ be a $k$-Lipschitzian and $\eta$-strongly monotone operator on $H$ with $k>0$ and $\eta>0$, and let $V$ be an L-Lipschitzian operator. Suppose that $\Omega=\bigcap_{i=1}^{\infty} \operatorname{Fix}\left(T_{i}\right)$ is nonempty. Suppose that $x_{1} \in H, 0<\mu<\frac{2 \eta}{k^{2}}$ and $0<\gamma<\frac{\tau}{L}$ with $\tau=\mu\left(\eta-\frac{1}{2} \mu k^{2}\right)$. Let $\beta_{0}=1,\left\{\alpha_{n}\right\}$ be a sequence in $(0,1]$, and let $\left\{\beta_{n}\right\}$ be a strictly decreasing sequence in $(0,1]$. If the following conditions are satisfied:

(i) $\lim _{n \rightarrow \infty} \alpha_{n}=0$;

(ii) $\sum_{n=1}^{\infty} \alpha_{n}=\infty$;

(iii) $\sum_{n=1}^{\infty}\left|\alpha_{n+1}-\alpha_{n}\right|<\infty$;

(iv) $\sum_{n=1}^{\infty}\left|\beta_{n+1}-\beta_{n}\right|<\infty$.

Then $\left\{x_{n}\right\}$ generated by (1.7) converges strongly to $\tilde{x} \in \Omega$, which solves the variational inequality

$$
\langle(\mu F-\gamma V) \tilde{x}, z-\tilde{x}\rangle \geq 0, \quad \forall z \in \Omega .
$$

Equivalently, we have $P_{\Omega}(I-\mu F+\gamma V) \tilde{x}=\tilde{x}$.

Proof We proceed with the following steps:

Step 1: First show that there exists $\tilde{x} \in \Omega$ such that $\tilde{x}=P_{\Omega}(I-\mu F+\gamma V) \tilde{x}$.

In fact, by Lemma 2.3, $\mu F-\gamma V$ is strongly monotone. So, the variational inequality (3.6) has only one solution. We set $\tilde{x} \in \Omega$ to indicate the unique solution of (3.6). The variational inequality (3.6) can be written as

$$
\langle(I-\mu F+\gamma V) \tilde{x}-\tilde{x}, z-\tilde{x}\rangle \leq 0, \quad \forall z \in \Omega .
$$

So, by Lemma 2.4 , it is equivalent to the fixed point equation

$$
P_{\Omega}(I-\mu F+\gamma V) \tilde{x}=\tilde{x} .
$$

Step 2: Now we show that $\left\{x_{n}\right\}$ is bounded.

Let $p \in \Omega$, then for every $i \in \mathbb{N}, T_{i} p=p$. Observe that

$$
\begin{aligned}
\left\|y_{n}-p\right\| & \leq \beta_{n}\left\|x_{n}-p\right\|+\sum_{i=1}^{n}\left(\beta_{i-1}-\beta_{i}\right)\left\|T_{i} x_{n}-p\right\| \\
& \leq \beta_{n}\left\|x_{n}-p\right\|+\sum_{i=1}^{n}\left(\beta_{i-1}-\beta_{i}\right)\left\|x_{n}-p\right\|=\left\|x_{n}-p\right\| .
\end{aligned}
$$


Thus it follows that

$$
\begin{aligned}
\left\|x_{n+1}-p\right\| & =\left\|\alpha_{n} \gamma V x_{n}+\left(I-\mu \alpha_{n} F\right) y_{n}-p\right\| \\
& =\left\|\alpha_{n}\left(\gamma V x_{n}-\mu F p\right)+\left(I-\mu \alpha_{n} F\right) y_{n}-\left(I-\mu \alpha_{n} F\right) p\right\| \\
& \leq\left(1-\alpha_{n} \tau\right)\left\|y_{n}-p\right\|+\alpha_{n}\left(\left\|\gamma V x_{n}-\gamma V p\right\|+\|\gamma V p-\mu F p\|\right) \\
& \leq\left(1-\alpha_{n} \tau\right)\left\|y_{n}-p\right\|+\alpha_{n} \gamma L\left\|x_{n}-p\right\|+\alpha_{n}\|\gamma V p-\mu F p\| \\
& \leq\left[1-\alpha_{n}(\tau-\gamma L)\right]\left\|x_{n}-p\right\|+\alpha_{n}(\tau-\gamma L) \frac{\|\gamma V p-\mu F p\|}{\tau-\gamma L} \\
& \leq \max \left\{\left\|x_{n}-p\right\|, \frac{\|\gamma V p-\mu F p\|}{\tau-\gamma L}\right\} \\
& \leq \cdots \leq \max \left\{\left\|x_{1}-p\right\|, \frac{\|\gamma V p-\mu F p\|}{\tau-\gamma L}\right\} .
\end{aligned}
$$

Therefore, $\left\{x_{n}\right\}$ is bounded. Hence we can obtain that $\left\{y_{n}\right\},\left\{T_{i} x_{n}\right\},\left\{F y_{n}\right\}$ and $\left\{V x_{n}\right\}$ are bounded.

Step 3: we show $\lim _{n \rightarrow \infty}\left\|x_{n+1}-x_{n}\right\|=0$.

We observe that

$$
\begin{aligned}
x_{n+1}-x_{n}= & \alpha_{n} \gamma V x_{n}+\left(I-\mu \alpha_{n} F\right) y_{n}-\alpha_{n-1} \gamma V x_{n-1}-\left(I-\mu \alpha_{n-1} F\right) y_{n-1} \\
= & \alpha_{n} \gamma\left(V x_{n}-V x_{n-1}\right)+\left(\alpha_{n}-\alpha_{n-1}\right) \gamma V x_{n-1} \\
& +\left(\left(I-\mu \alpha_{n} F\right) y_{n}-\left(I-\mu \alpha_{n} F\right) y_{n-1}\right)+\left(\alpha_{n-1}-\alpha_{n}\right) \mu F y_{n-1} .
\end{aligned}
$$

It follows that

$$
\begin{aligned}
\left\|x_{n+1}-x_{n}\right\| \leq & \alpha_{n} \gamma\left\|V x_{n}-V x_{n-1}\right\|+\left|\alpha_{n}-\alpha_{n-1}\right| \gamma\left\|V x_{n-1}\right\| \\
& +\left(1-\alpha_{n} \tau\right)\left\|y_{n}-y_{n-1}\right\|+\left|\alpha_{n-1}-\alpha_{n}\right| \mu\left\|F y_{n-1}\right\| .
\end{aligned}
$$

We have

$$
\begin{aligned}
\left\|y_{n}-y_{n-1}\right\|= & \left\|\beta_{n} x_{n}+\sum_{i=1}^{n}\left(\beta_{i-1}-\beta_{i}\right) T_{i} x_{n}-\beta_{n-1} x_{n-1}-\sum_{i=1}^{n-1}\left(\beta_{i-1}-\beta_{i}\right) T_{i} x_{n-1}\right\| \\
\leq & \beta_{n}\left\|x_{n}-x_{n-1}\right\|+\left|\beta_{n}-\beta_{n-1}\right|\left\|x_{n-1}\right\| \\
& +\sum_{i=1}^{n}\left(\beta_{i-1}-\beta_{i}\right)\left\|T_{i} x_{n}-T_{i} x_{n-1}\right\|+\left|\beta_{n}-\beta_{n-1}\right|\left\|T_{n} x_{n-1}\right\| \\
\leq & \left\|x_{n}-x_{n-1}\right\|+\left|\beta_{n}-\beta_{n-1}\right|\left(\left\|x_{n-1}\right\|+\left\|T_{n} x_{n-1}\right\|\right) .
\end{aligned}
$$

Combining (3.7) and (3.8), we obtain that

$$
\begin{aligned}
\left\|x_{n+1}-x_{n}\right\| \leq & \alpha_{n} \gamma L\left\|x_{n}-x_{n-1}\right\|+\left|\alpha_{n}-\alpha_{n-1}\right|\left(\gamma\left\|V x_{n-1}\right\|+\mu\left\|F y_{n-1}\right\|\right) \\
& +\left(1-\alpha_{n} \tau\right)\left\|x_{n}-x_{n-1}\right\|+\left|\beta_{n}-\beta_{n-1}\right|\left(1-\alpha_{n} \tau\right)\left(\left\|x_{n-1}\right\|+\left\|T_{n} x_{n-1}\right\|\right) \\
= & \left(1-\alpha_{n}(\tau-\gamma L)\right)\left\|x_{n}-x_{n-1}\right\|+\left|\alpha_{n}-\alpha_{n-1}\right|\left(\gamma\left\|V x_{n-1}\right\|+\mu\left\|F y_{n-1}\right\|\right) \\
& +\left|\beta_{n}-\beta_{n-1}\right|\left(1-\alpha_{n} \tau\right)\left(\left\|x_{n-1}\right\|+\left\|T_{n} x_{n-1}\right\|\right) .
\end{aligned}
$$

Using (ii), (iii), (iv) and Lemma 2.2, we have $\lim _{n \rightarrow \infty}\left\|x_{n+1}-x_{n}\right\|=0$. 
Step 4: We show $\lim _{n \rightarrow \infty}\left\|T_{i} x_{n}-x_{n}\right\|=0$ for all $i \in \mathbb{N}$.

Since $p \in \Omega$, we note that

$$
\begin{aligned}
\left\|x_{n}-p\right\|^{2} & \geq\left\|T_{i} x_{n}-T_{i} p\right\|^{2}=\left\|T_{i} x_{n}-x_{n}+x_{n}-p\right\|^{2} \\
& =\left\|T_{i} x_{n}-x_{n}\right\|^{2}+\left\|x_{n}-p\right\|^{2}+2\left\langle T_{i} x_{n}-x_{n}, x_{n}-p\right\rangle,
\end{aligned}
$$

which implies that

$$
\frac{1}{2}\left\|T_{i} x_{n}-x_{n}\right\|^{2} \leq\left\langle x_{n}-T_{i} x_{n}, x_{n}-p\right\rangle .
$$

From (1.7) and (3.9), we deduce

$$
\begin{aligned}
\frac{1}{2} \sum_{i=1}^{n}\left(\beta_{i-1}-\beta_{i}\right)\left\|T_{i} x_{n}-x_{n}\right\|^{2} & \leq \sum_{i=1}^{n}\left(\beta_{i-1}-\beta_{i}\right)\left\langle x_{n}-T_{i} x_{n}, x_{n}-p\right\rangle \\
& =\left\langle\left(1-\beta_{n}\right) x_{n}-\sum_{i=1}^{n}\left(\beta_{i-1}-\beta_{i}\right) T_{i} x_{n}, x_{n}-p\right\rangle \\
& =\left\langle\left(1-\beta_{n}\right) x_{n}-y_{n}+\beta_{n} x_{n}, x_{n}-p\right\rangle \\
& =\left\langle x_{n}-y_{n}, x_{n}-p\right\rangle \\
& =\left\langle x_{n}-x_{n+1}, x_{n}-p\right\rangle+\left\langle x_{n+1}-y_{n}, x_{n}-p\right\rangle .
\end{aligned}
$$

Using (1.7), we can have

$$
\begin{aligned}
\frac{1}{2} \sum_{i=1}^{n}\left(\beta_{i-1}-\beta_{i}\right)\left\|T_{i} x_{n}-x_{n}\right\|^{2} & \leq\left\langle x_{n}-x_{n+1}, x_{n}-p\right\rangle+\alpha_{n}\left\langle\gamma V x_{n}-\mu F y_{n}, x_{n}-p\right\rangle \\
& \leq\left\|x_{n}-x_{n+1}\right\|\left\|x_{n}-p\right\|+\alpha_{n}\left\|\gamma V x_{n}-\mu F y_{n}\right\|\left\|x_{n}-p\right\| .
\end{aligned}
$$

Noting that $\lim _{n \rightarrow \infty}\left\|x_{n}-x_{n+1}\right\|=0$ and $\lim _{n \rightarrow \infty} \alpha_{n}=0$, we immediately obtain

$$
\lim _{n \rightarrow \infty} \sum_{i=1}^{n}\left(\beta_{i-1}-\beta_{i}\right)\left\|T_{i} x_{n}-x_{n}\right\|^{2}=0 .
$$

Since $\left\{\beta_{n}\right\}$ is strictly decreasing, it follows that for every $i \in \mathbb{N}$,

$$
\lim _{n \rightarrow \infty}\left\|T_{i} x_{n}-x_{n}\right\|=0 .
$$

Step 5: Show $\lim \sup _{n \rightarrow \infty}\left\langle(\gamma V-\mu F) \tilde{x}, x_{n}-\tilde{x}\right\rangle \leq 0$, where $\tilde{x}=P_{\Omega}(I-\mu F+\gamma V) \tilde{x}$.

Since $\left\{x_{n}\right\}$ is bounded, there exist a point $v \in H$ and a subsequence $\left\{x_{n_{k}}\right\}$ of $\left\{x_{n}\right\}$ such that

$$
\limsup _{n \rightarrow \infty}\left\langle(\gamma V-\mu F) \tilde{x}, x_{n}-\tilde{x}\right)=\lim _{k \rightarrow \infty}\left\langle(\gamma V-\mu F) \tilde{x}, x_{n_{k}}-\tilde{x}\right\rangle
$$

and $x_{n_{k}} \rightarrow v$. Now, applying (3.10) and Lemma 2.1, we conclude that $v \in \operatorname{Fix}\left(T_{i}\right)$ for every $i \in \mathbb{N}$. Hence, $v \in \Omega$. Since $\Omega$ is closed and convex, by Lemma 2.4 , we get

$$
\begin{aligned}
\limsup _{n \rightarrow \infty}\left\langle(\gamma V-\mu F) \tilde{x}, x_{n}-\tilde{x}\right\rangle & =\lim _{k \rightarrow \infty}\left\langle(\gamma V-\mu F) \tilde{x}, x_{n_{k}}-\tilde{x}\right\rangle \\
& =\langle(\gamma V-\mu F) \tilde{x}, \nu-\tilde{x}\rangle \leq 0 .
\end{aligned}
$$


Step 6: Show $x_{n} \rightarrow \tilde{x}=P_{\Omega}(I-\mu F+\gamma V)(\tilde{x})$.

Since $\tilde{x} \in \Omega$, we have $T_{i} \tilde{x}=\tilde{x}$ for every $i \in \mathbb{N}$. Using (1.7), we have

$$
\begin{aligned}
\left\|x_{n+1}-\tilde{x}\right\|^{2}= & \left\|\alpha_{n} \gamma V x_{n}+\left(I-\mu \alpha_{n} F\right) y_{n}-\tilde{x}\right\|^{2} \\
= & \left\|\left(I-\mu \alpha_{n} F\right) y_{n}-\left(I-\mu \alpha_{n} F\right) \tilde{x}+\alpha_{n}\left(\gamma V x_{n}-\mu F \tilde{x}\right)\right\|^{2} \\
\leq & \left\|\left(I-\mu \alpha_{n} F\right) y_{n}-\left(I-\mu \alpha_{n} F\right) \tilde{x}\right\|^{2}+2 \alpha_{n}\left\langle\gamma V x_{n}-\mu F \tilde{x}, x_{n+1}-\tilde{x}\right\rangle \\
\leq & \left(1-\alpha_{n} \tau\right)^{2}\left\|y_{n}-\tilde{x}\right\|^{2}+2 \alpha_{n} \gamma\left\langle V x_{n}-V \tilde{x}, x_{n+1}-\tilde{x}\right\rangle \\
& +2 \alpha_{n}\left\langle\gamma V \tilde{x}-\mu F \tilde{x}, x_{n+1}-\tilde{x}\right\rangle \\
\leq & \left(1-\alpha_{n} \tau\right)^{2}\left\|x_{n}-\tilde{x}\right\|^{2}+\alpha_{n} L \gamma\left(\left\|x_{n}-\tilde{x}\right\|^{2}+\left\|x_{n+1}-\tilde{x}\right\|^{2}\right) \\
& +2 \alpha_{n}\left\langle\gamma V \tilde{x}-\mu F \tilde{x}, x_{n+1}-\tilde{x}\right\rangle,
\end{aligned}
$$

which implies that

$$
\begin{aligned}
\left\|x_{n+1}-\tilde{x}\right\|^{2} \leq & \frac{\left(1-\alpha_{n} \tau\right)^{2}+\alpha_{n} \gamma L}{1-\alpha_{n} \gamma L}\left\|x_{n}-\tilde{x}\right\|^{2}+\frac{2 \alpha_{n}}{1-\alpha_{n} \gamma L}\left\langle\gamma V \tilde{x}-\mu F \tilde{x}, x_{n+1}-\tilde{x}\right\rangle \\
\leq & \left(1-\frac{2 \alpha_{n}(\tau-\gamma L)}{1-\alpha_{n} \gamma L}\right)\left\|x_{n}-\tilde{x}\right\|^{2} \\
& +\frac{2 \alpha_{n}(\tau-\gamma L)}{1-\alpha_{n} \gamma L}\left[\frac{1}{\tau-\gamma L}\left\langle\gamma V \tilde{x}-\mu F \tilde{x}, x_{n+1}-\tilde{x}\right\rangle\right] .
\end{aligned}
$$

Consequently, according to (3.11) and Lemma 2.2, we deduce that $\left\{x_{n}\right\}$ converges strongly to $\tilde{x}=P_{\Omega}(I-\mu F+\gamma V) \tilde{x}$. This completes the proof.

Corollary 3.4 Let $T$ be a nonexpansive self-mapping of a real Hilbert space $H$, let $F$ be a $k$-Lipschitzian and $\eta$-strongly monotone operator on $H$ with $k>0$ and $\eta>0$, and let $V$ be an L-Lipschitzian operator. Suppose that $\Omega=\operatorname{Fix}(T)$ is nonempty. Suppose that $x_{1} \in H$ and that $\left\{x_{n}\right\}$ is generated by the following algorithm:

$$
x_{n+1}=\alpha_{n} \gamma V\left(x_{n}\right)+\left(I-\mu \alpha_{n} F\right) T x_{n},
$$

where $0<\mu<\frac{2 \eta}{k^{2}}$ and $0<\gamma<\frac{\tau}{L}$ with $\tau=\mu\left(\eta-\frac{1}{2} \mu k^{2}\right)$. Let $\left\{\alpha_{n}\right\}$ be in $(0,1]$. If the following conditions are satisfied:

(i) $\lim _{n \rightarrow \infty} \alpha_{n}=0$;

(ii) $\sum_{n=1}^{\infty} \alpha_{n}=\infty$;

(iii) $\sum_{n=1}^{\infty}\left|\alpha_{n+1}-\alpha_{n}\right|<\infty$.

Then $\left\{x_{n}\right\}$ converges strongly to $\tilde{x} \in \Omega$, which solves the variational inequality

$$
\langle(\mu F-\gamma V) \tilde{x}, z-\tilde{x}\rangle \geq 0, \quad \forall z \in \Omega
$$

Equivalently, we have $P_{\Omega}(I-\mu F+\gamma V) \tilde{x}=\tilde{x}$.

Proof Set $\left\{T_{n}\right\}$ to be the sequences of operators defined by $T_{n}=T$ for all $n \in \mathbb{N}$ in Theorem 3.3. Then by Theorem 3.3, we obtain the desired result. 


\section{Application in the split common fixed point problem}

Let $H_{1}$ and $H_{2}$ be Hilbert spaces, let $A: H_{1} \rightarrow H_{2}$ be a bounded linear operator. The split common fixed point problem (SCFPP) is to find a point $x^{*} \in H_{1}$ satisfied with

$$
x^{*} \in \bigcap_{i=1}^{p} \operatorname{Fix}\left(U_{i}\right), \quad A x^{*} \in \bigcap_{j=1}^{r} \operatorname{Fix}\left(T_{j}\right),
$$

where $U_{i}: H_{1} \rightarrow H_{1}(i=1,2, \ldots, p)$ and $T_{j}: H_{2} \rightarrow H_{2}(j=1,2, \ldots, r)$ are nonlinear operators. The concept of the SCFPP in finite-dimensional Hilbert spaces was firstly introduced by Censor and Segal in [35]. Now we consider a generalized split common fixed point problem (GSCFPP) which is to find a point

$$
x^{*} \in \bigcap_{i=1}^{\infty} \operatorname{Fix}\left(U_{i}\right), \quad A x^{*} \in \bigcap_{j=1}^{\infty} \operatorname{Fix}\left(T_{j}\right) .
$$

We know that if for all $i$ and $j, U_{i}$ and $T_{j}$ are nonexpansive operators, the GSCFPP is equivalent to the following common fixed point problem:

$$
x^{*} \in \bigcap_{i=1}^{\infty} \operatorname{Fix}\left(U_{i}\right), \quad x^{*} \in \bigcap_{j=1}^{\infty} \operatorname{Fix}\left(V_{j}\right),
$$

where $V_{j}=I-\gamma A^{*}\left(I-T_{j}\right) A$ with $0<\gamma \leq \frac{1}{\|A\|^{2}}$ for every $j \in \mathbb{N}$ (see [36]). The solution set of GSCFPP (4.1) is denoted by $S$.

Theorem 4.1 Let $\left\{U_{n}\right\}$ and $\left\{T_{n}\right\}$ be sequences of nonexpansive operators on real Hilbert spaces $H_{1}$ and $H_{2}$, respectively. Let $F$ be a $k$-Lipschitzian and $\eta$-strongly monotone operator on $H_{1}$ with $k>0$ and $\eta>0$. Let $V$ be an L-Lipschitzian operator. Suppose that $S$ is nonempty. Suppose that $x_{1} \in H$ and that $\left\{x_{n}\right\}$ is generated by the following algorithm:

$$
\left\{\begin{array}{l}
y_{n}=\beta_{n} x_{n}+\sum_{i=1}^{n}\left(\beta_{i-1}-\beta_{i}\right) U_{i}\left(I-\gamma A^{*}\left(I-T_{i}\right) A\right) x_{n} \\
x_{n+1}=\alpha_{n} \gamma V\left(x_{n}\right)+\left(I-\mu \alpha_{n} F\right) y_{n}
\end{array}\right.
$$

where $0<\mu<\frac{2 \eta}{k^{2}}$ and $0<\gamma<\frac{\tau}{L}$ with $\tau=\mu\left(\eta-\frac{1}{2} \mu k^{2}\right)$. Let $\left\{\alpha_{n}\right\}$ be in $(0,1],\left\{\beta_{n}\right\}$ be a strictly decreasing sequence in $(0,1]$ and $\beta_{0}=1$. If the following conditions are satisfied:

(i) $\lim _{n \rightarrow \infty} \alpha_{n}=0$;

(ii) $\sum_{n=1}^{\infty} \alpha_{n}=\infty$;

(iii) $\sum_{n=1}^{\infty}\left|\alpha_{n+1}-\alpha_{n}\right|<\infty$;

(iv) $\sum_{n=1}^{\infty}\left|\beta_{n+1}-\beta_{n}\right|<\infty$.

Then $\left\{x_{n}\right\}$ converges strongly to $\tilde{x} \in S$, which solves the variational inequality

$$
\langle(\mu F-\gamma V) \tilde{x}, z-\tilde{x}\rangle \geq 0, \quad \forall z \in S
$$

Equivalently, we have $P_{\Omega}(I-\mu F+\gamma V) \tilde{x}=\tilde{x}$.

Proof Set $\left\{T_{n}\right\}$ to be the sequences of operators defined by $T_{n}:=U_{n}\left(I-\gamma A^{*}\left(I-T_{n}\right) A\right)$ for all $n \in \mathbb{N}$ in Theorem 3.3. By Theorem 3.3, we can obtain

$$
\lim _{n \rightarrow \infty}\left\|U_{i}\left(I-\gamma A^{*}\left(I-T_{i}\right) A\right) x_{n}-x_{n}\right\|=0
$$


in Step 4. But it does not imply that the set of cluster points of the weak topology $\omega_{w}\left(x_{n}\right)$ is a subset of $S$. In order to prove this, we only show $\lim _{n \rightarrow \infty}\left\|T_{i} A x_{n}-x_{n}\right\|=0$ and $\lim _{n \rightarrow \infty}\left\|U_{i} x_{n}-x_{n}\right\|=0$.

Since $p \in \Omega, T_{i} A p=A p$. Hence, for every $i \in \mathbb{N}$,

$$
\begin{aligned}
\left\|A x_{n}-A p\right\|^{2} & \geq\left\|T_{i} A x_{n}-T_{i} A p\right\|^{2}=\left\|T_{i} A x_{n}-A p\right\|^{2} \\
& =\left\|T_{i} A x_{n}-A x_{n}+A x_{n}-A p\right\|^{2} \\
& =\left\|T_{i} A x_{n}-A x_{n}\right\|^{2}+\left\|A x_{n}-A p\right\|^{2}+2\left\langle T_{i} A x_{n}-A x_{n}, A x_{n}-A p\right\rangle,
\end{aligned}
$$

which yields that

$$
\left\langle T_{i} A x_{n}-A x_{n}, A x_{n}-A p\right\rangle \leq-\frac{1}{2}\left\|T_{i} A x_{n}-A x_{n}\right\|^{2}
$$

for every $i \in \mathbb{N}$. Using (4.2), we note that

$$
\begin{aligned}
\left\|y_{n}-p\right\|^{2}= & \left\|\beta_{n}\left(x_{n}-p\right)+\sum_{i=1}^{n}\left(\beta_{i-1}-\beta_{i}\right)\left(U_{i}\left(I-\gamma A^{*}\left(I-T_{i}\right) A\right) x_{n}-p\right)\right\|^{2} \\
\leq & \beta_{n}\left\|x_{n}-p\right\|^{2}+\sum_{i=1}^{n}\left(\beta_{i-1}-\beta_{i}\right)\left\|x_{n}+\gamma A^{*}\left(T_{i}-I\right) A x_{n}-p\right\|^{2} \\
\leq & \beta_{n}\left\|x_{n}-p\right\|^{2}+\sum_{i=1}^{n}\left(\beta_{i-1}-\beta_{i}\right)\left(\left\|x_{n}-p\right\|^{2}+\gamma^{2}\|A\|^{2}\left\|T_{i} A x_{n}-A x_{n}\right\|^{2}\right. \\
& \left.+2 \gamma\left\langle A x_{n}-A p, T_{i} A x_{n}-A x_{n}\right\rangle\right) \\
\leq & \left\|x_{n}-p\right\|^{2}+\sum_{i=1}^{n}\left(\beta_{i-1}-\beta_{i}\right)\left(\gamma^{2}\|A\|^{2}\left\|T_{i} A x_{n}-A x_{n}\right\|^{2}\right. \\
& \left.-\gamma\left\|T_{i} A x_{n}-A x_{n}\right\|^{2}\right) \\
= & \left\|x_{n}-p\right\|^{2}+\gamma\left(\gamma\|A\|^{2}-1\right) \sum_{i=1}^{n}\left(\beta_{i-1}-\beta_{i}\right)\left\|T_{i} A x_{n}-A x_{n}\right\|^{2} .
\end{aligned}
$$

Thus

$$
\begin{aligned}
\gamma & \left(1-\gamma\|A\|^{2}\right) \sum_{i=1}^{n}\left(\beta_{i-1}-\beta_{i}\right)\left\|T_{i} A x_{n}-A x_{n}\right\|^{2} \\
& =\left\|x_{n}-p\right\|^{2}-\left\|y_{n}-p\right\|^{2} \\
& =\left(\left\|x_{n}-p\right\|-\left\|y_{n}-p\right\|\right)\left(\left\|x_{n}-p\right\|+\left\|y_{n}-p\right\|\right) \\
& \leq\left\|x_{n}-y_{n}\right\|\left(\left\|x_{n}-p\right\|+\left\|y_{n}-p\right\|\right) .
\end{aligned}
$$

It follows that $\lim _{n \rightarrow \infty}\left\|T_{i} A x_{n}-A x_{n}\right\|=0$. Now we show that $\lim _{n \rightarrow \infty}\left\|U_{i} x_{n}-x_{n}\right\|=0$. Note that

$$
\begin{aligned}
\left\|U_{i} x_{n}-x_{n}\right\| & \leq\left\|U_{i} x_{n}-U_{i}\left(I-\gamma A^{*}\left(I-T_{i}\right) A\right) x_{n}\right\|+\left\|U_{i}\left(I-\gamma A^{*}\left(I-T_{i}\right) A\right) x_{n}-x_{n}\right\| \\
& \leq\left\|x_{n}-\left(I-\gamma A^{*}\left(I-T_{i}\right) A\right) x_{n}\right\|+\left\|U_{i}\left(I-\gamma A^{*}\left(I-T_{i}\right) A\right) x_{n}-x_{n}\right\| \\
& \leq \gamma\|A\|\left\|\left(T_{i}-I\right) A x_{n}\right\|+\left\|U_{i}\left(I-\gamma A^{*}\left(I-T_{i}\right) A\right) x_{n}-x_{n}\right\| .
\end{aligned}
$$


Then we have $\lim _{n \rightarrow \infty}\left\|U_{i} x_{n}-x_{n}\right\|=0$ for every $i \in \mathbb{N}$. Then we can have $\omega_{w}\left(x_{n}\right) \subset S$. Hence, by Theorem 3.3, we obtain the desired result.

\author{
Competing interests \\ The authors declare that they have no competing interests.
}

Authors' contributions

All authors contributed equally and significantly in writing this article. All authors read and approved the final manuscript.

\title{
Acknowledgements
}

This research is supported by the Fundamental Science Research Funds for the Central Universities (Program No. ZXH2012K001).

Received: 5 December 2012 Accepted: 9 May 2013 Published: 29 May 2013

\section{References}

1. Moudafi, A: Viscosity approximation methods for fixed-points problems. J. Math. Anal. Appl. 241, 46-55 (2000)

2. Xu, HK: Viscosity approximation methods for nonexpansive mapping. J. Math. Anal. Appl. 298, $279-291$ (2004)

3. Xu, HK: An iterative approach to quadratic optimization. J. Optim. Theory Appl. 116, 659-678 (2003)

4. Yamada, l: The hybrid steepest descent for the variational inequality problems over the intersection of fixed points sets of nonexpansive mappings. In: Inherently Parallel Algorithms in Feasibility and Optimization and Their Applications. Studies in Computational Mathematics, vol. 8, pp. 473-504 (2001)

5. Marino, G, Xu, HK: A general iterative method for nonexpansive mappings in Hilbert spaces. J. Math. Anal. Appl. 318 , 43-52 (2006)

6. Tian, M: A general iterative algorithm for nonexpansive mappings in Hilbert spaces. Nonlinear Anal. 73, 689-694 (2010)

7. Tian, M, Di, LY: A general iterative method based on the hybrid steepest descent scheme for variational inclusions, equilibrium problems. J. Appl. Math. Inf. 29, 603-619 (2011)

8. Wang, S: A general iterative method for an infinite family of strictly pseudo-contractive mappings in Hilbert spaces. Appl. Math. Lett. 24(6), 901-907 (2010)

9. Ceng, LC, Ansari, QH, Yao, JC: Some iterative methods for finding fixed points and for solving constrained convex minimization problems. Nonlinear Anal. 74, 5286-5302 (2011)

10. Ceng, LC, Guu, SM, Yao, JC: A general composite iterative algorithm for nonexpansive mappings in Hilbert spaces. Comput. Math. Appl. 61(9), 2447-2455 (2011)

11. Jung, JS: Some results on a general iterative method for $k$-strictly pseudo-contractive mappings. Fixed Point Theory Appl. 2011, 24 (2011). doi:10.1186/1687-1812-2011-24

12. Piri, $\mathrm{H}$, Badali, $\mathrm{AH}$ : Strong convergence theorem for amenable semigroups of nonexpansive mappings and variational inequalities. Fixed Point Theory Appl. 2011, 55 (2011). doi:10.1186/1687-1812-2011-55

13. Piri, $\mathrm{H}$ : A general iterative method for finding common solutions of system of equilibrium problems, system of variational inequalities and fixed point problems. Math. Comput. Model. 55(3-4), 1622-1638 (2012)

14. Sunthrayuth, $P$, Kumam, P: Iterative methods for variational inequality problems and fixed point problems of a countable family of strict pseudo-contractions in a q-uniformly smooth Banach space. Fixed Point Theory Appl. 2012, 65 (2012). doi:10.1186/1687-1812-2012-65

15. Jung, JS: Some algorithms for finding fixed points and solutions of variational inequalities. Abstr. Appl. Anal. 2012, Article ID 153456 (2012)

16. Sunthrayuth, $P$, Kumam, $P$ : Iterative algorithms approach to a general system of nonlinear variational inequalities with perturbed mappings and fixed point problems for nonexpansive semigroups. J. Inequal. Appl. 2012, 133 (2012)

17. Tian, M, Jin, X: Strong convergent result for quasi-nonexpansive mappings in Hilbert spaces. Fixed Point Theory Appl. 2011, 88 (2011). doi:10.1186/1687-1812-2011-88

18. Duan, PC, Wang, AH: General iterative methods for equilibrium problems and infinitely many strict pseudo-contractions in Hilbert spaces. J. Appl. Math. 2012, Article ID 602513 (2012)

19. Zegeye, $\mathrm{H}$, Shahzad, $\mathrm{N}$ : Approximation of the common minimum-norm fixed point of a finite family of asymptotically nonexpansive mapping. Fixed Point Theory Appl. 2013, 1 (2013)

20. Zegeye, H, Shahzad, N: Strong convergence of an iterative method for pseudo-contractive and monotone mappings. J. Glob. Optim. 54(1), 173-184 (2012)

21. Zegeye, $\mathrm{H}$, Shahzad, $\mathrm{N}$ : Approximating common solution of variational inequality problems for two monotone mappings in Banach spaces. Optim. Lett. 5(4), 691-704 (2011)

22. Zegeye, $\mathrm{H}$, Shahzad, N: Strong convergence theorems for monotone mappings and relatively weak nonexpansive mappings. Nonlinear Anal. 70(7), 2707-2716 (2009)

23. Yao, Y, Liou, YC, Shahzad, N: A strongly convergent method for the split feasibility problem. Abstr. Appl. Anal. 2012, Article ID 125046 (2012)

24. Yao, Y, Cho, YJ, Chen, R: An iterative algorithm for solving fixed point problems, variational inequality problems and mixed equilibrium problems. Nonlinear Anal., Theory Methods Appl. 71, 3363-3373 (2009)

25. Colaoa, V, Acedob, GL, Marinoa, G: An implicit method for finding common solutions of variational inequalities and systems of equilibrium problems and fixed points of infinite family of nonexpansive mappings. Nonlinear Anal., Theory Methods Appl. 71, 2708-2715 (2009)

26. Yao, Y, Liou, Y, Yao, J: An iterative algorithm for approximating convex minimization problem. Appl. Math. Comput. $188,648-656(2007)$ 
27. Yao, Y, Cho, YJ, Liou, Y: Algorithms of common solutions for variational inclusions, mixed equilibrium problems and fixed point problems. Eur. J. Oper. Res. 212, 242-250 (2011)

28. Yao, Y, Liou, Y, Kang, SM: Strong convergence of an iterative algorithm on an infinite countable family of nonexpansive mappings. Appl. Math. Comput. 208, 211-218 (2009)

29. Yao, Y, Liou, Y, Chen, R: A general iterative method for an infinite family of nonexpansive mappings. Nonlinear Anal., Theory Methods Appl. 69, 1644-1654 (2008)

30. Yao, Y, Noor, MA, Liou, Y: A new hybrid iterative algorithm for variational inequalities. Appl. Math. Comput. 216, 822-829 (2010)

31. Yao, Y, Liou, Y, Yao, J: A new hybrid iterative algorithm for fixed-point problems, variational inequality problems, and mixed equilibrium problems. Fixed Point Theory Appl. 2008, Article ID 417089 (2008)

32. Goebel, K, Kirk, WA: Topics in Metric Fixed-Point Theory. Cambridge University Press, Cambridge (1990)

33. Tian, M: A general iterative method based on the hybrid steepest descent scheme for nonexpansive mappings in Hilbert spaces. In: International Conference on Computational Intelligence and Software Engineering (CiSE) (2010)

34. Marino, G, Xu, HK: Weak and strong convergence theorems for strict pseudo-contractions in Hilbert spaces. J. Math. Anal. Appl. 329, 336-346 (2007)

35. Censor, Y, Segal, A: The split common fixed point problem for directed operators. J. Convex Anal. 19, 587-600 (2009)

36. Zhang, C: Strong convergence theorem for the generalized split common fixed point problem. J. Appl. Math. 2012 Article ID 575014 (2012)

doi:10.1186/1687-1812-2013-138

Cite this article as: Zhang and He: A general iterative algorithm for an infinite family of nonexpansive operators in Hilbert spaces. Fixed Point Theory and Applications 2013 2013:138.

\section{Submit your manuscript to a SpringerOpen ${ }^{\circ}$ journal and benefit from:}

- Convenient online submission

- Rigorous peer review

- Immediate publication on acceptance

- Open access: articles freely available online

- High visibility within the field

- Retaining the copyright to your article 